\title{
Volterra Runge- Kutta Methods for Solving Nonlinear Volterra Integral Equations
}

\author{
Muna M. Mustafa*
}

Received 17, March, 2009

Acceptance 22, June, 2009

\begin{abstract}
:
In this paper Volterra Runge-Kutta methods which include: method of order two and four will be applied to general nonlinear Volterra integral equations of the second kind. Moreover we study the convergent of the algorithms of Volterra RungeKutta methods.

Finally, programs for each method are written in MATLAB language and a comparison between the two types has been made depending on the least square errors.
\end{abstract}

Key words: nonlinear Volterra integral equations, Volterra Runge-Kutta method.

\section{Introduction:}

Volterra integral equations (VIEs) are the mathematical model of many evolutionary problems with memory arising from biology, chemistry, physics, engineering. For example they arise from population dynamics, epidemic diffusion, neurophysiology, and feedback control theory, study of the behavior of nuclear reactors and from the treatment of special hyperbolic differential equations [1].

In recent year, numerous works

have been focusing on the development of more advanced and efficient methods for integral equations such as computational methods[2], linearization method[3], fast RungeKutta methods for nonlinear Volterra integral equations of convolution type[4] and collocation solutions of a weakly singular VIE[5]. In this paper we construct numerical methods for nonlinear VIEs of the second kind based on Volterra Runge-Kutta methods which produce accurate solution at a low computational cost and catch the qualitative behavior of the exact solution.

\section{Theoretical background:}

An integral equation is a functional in which the unknown function appears under one or several integral signs. In this paper we focus our attention on Volterra Integral Equations (VIEs) characterized by a variable upper limit of integration.

Let $\mathrm{I}=[0, \mathrm{~T}]$ denote a given closed and bounded interval, with $\mathrm{T}>0$, and set $\mathrm{S}=\{(\mathrm{t}, \mathrm{s}) \mid 0 \leq s \leq t \leq T\}$. The more general VIE(for the unknown function $y)$ is of the form[1]:

$$
\begin{gathered}
\theta(t) y(t)=f(t)+ \\
\int_{0}^{t} k(t, s, y(s)) d s, t \in I
\end{gathered}
$$

$\theta, f, y: I \rightarrow R, k: S \times R \rightarrow R$ where the function $f$ is referred to as forcing function and $k$ is called the kernel of the integral equation. The function $\theta(t)$ determines the classification of VIEs in:

\footnotetext{
*Department of Mathematics, College of Science for Women, Baghdad University,
} Al-Jadriyah, Baghdad, Iraq 
- first kind VIEs. If $\theta(t)=0, \forall t \in I$, the equation (1) becomes:

$$
f(t)=-\int_{0}^{t} k(t, s, y(s)) d s, t \in I
$$

- second kind VIEs. If $\theta(t)=1, \forall t \in$ $I$, the equation (1) can be led to the form:

$$
y(t)=f(t)+\int_{0}^{t} k(t, s, y(s)) d s, t \in
$$

- third kind VIEs. If $\theta$ is a continuous function possessing a finite number of zeros in the interval I.

In this paper we focus our attention on second kind VIEs.

A VIE is said to be:

linear if its kernel has the form: $k(t, s, y)=\bar{k}(t, s) y, \forall t, s, y$

of Hammerstein type if: $k(t, s, y)=$ $\bar{k}(t, s) g(s, y(s))$, where $\mathrm{g}: I \rightarrow R$ is continuous function.

weakly singular (or of Able type) if $k(t, s, y)=(t-s)^{-\alpha} \gamma(t, s, y), 0<$

$\alpha<1$, where $\gamma$ is a smooth function in $S \times R$.

In case of the general second kind integral equation(2) we report two (global and local) existence and uniqueness theorems under the assumption that the kernel $k$ satisfies a uniform or punctual Lipschitz condition.

\section{Theorem(1): [1]}

Let $k(t, s, y)$ be continuous for all $(t, s) \in S$ and all $y$, and suppose that $k$ satisfy the uniform Lipschitz condition:

$$
\left|k\left(t, s, y_{1}\right)-k\left(t, s, y_{2}\right)\right| \leq
$$

$L\left|y_{1}-y_{2}\right|, \forall(t, s) \in S, \forall y_{1}, y_{2} \in R$

with Lipschitz constant $L$ being independent of $y_{1}, y_{2}$. Then for each $f \in C(I)$ the nonlinear equation(2.2) possesses a unique solution $y \in C(I)$.

Theorem(2):[1]

Let $f \in C(I)$ and assume that $k(t, s, y)$ is continuous in the region:

$S,|y-f(t)| \leq B\}$
In addiction let $k$ satisfy a Lipschitz condition of the form:

$$
\left|k\left(t, s, y_{1}\right)-k\left(t, s, y_{2}\right)\right| \leq
$$

$L\left|y_{1}-y_{2}\right|, \forall\left(t, s, y_{1}\right),\left(t, s, y_{2}\right) \in \pi$ and set $M=\max \{k(t, s, y) \mid(t, s, y) \in$ $\pi\}$ and $T_{0}=\min \left\{T, \frac{B}{M}\right\}$. Then the nonlinear equation (2) possesses a unique continuous solution $y$ in $\left[0, T_{0}\right]$.

\section{Volterra Runge- Kutta methods:}

Runge-Kutta method is based on a discretization of the given interval $I=[0, T]$ by a uniform mesh:

$$
I_{h}=\left\{t_{n}=n h, n=\right.
$$

$\left.0, \ldots, N_{t}, h \geq 0, N_{t}=T\right\}$

The integral equation (2) can be rewritten, by relating it to this mish, as[6]:

$y(t)=F_{n}(t)+\emptyset_{n}(t)$

where

$F_{n}(t)=f(t)+\int_{0}^{t_{n}} k(t, s, y(s)) d s$

...(5)

and

$\emptyset_{n}(t)=\int_{t_{n}}^{t} k(t, s, y(s)) d s$

represent respectively the lag term and the increment function. The lag term or tail term contains the past history of the phenomenon. Since it depends on time $t$, it has to be computed for each time step and its cost increases when time passes. Thus the lag term computation makes the numerical integration of VIEs very expensive in terms of computational cost[1].

Runge-Kutta methods for the numerical solution of VIEs were introduced by Pouzet and Bel'tyukov about the mid-1960.

A Volterra Runge-Kutta (VRK) method is based on an approximation scheme for the increment function(6) that will be called a VRK formula and denoted by $\bar{\emptyset}_{n}(t)$, and on an approximation scheme, $\bar{F}_{n}(t)$, for the lag term (5) that will be called lag term formula. 
The approximation of the equation in the mesh point $t_{n+1}$ leads to the discrete method of the form:

$y_{n+1}=\bar{F}_{n}\left(t_{n}+h\right)+$ $\bar{\emptyset}_{n}\left(t_{n}+h\right), n=0, \ldots, N_{t}-1$ ...(7)

In this paper we will consider extended VRK methods of Pouzet type.

\section{(1) Extended VRK methods of Pouzet type:}

An extended VRK method of pouzet type (PVRK method) uses an m-stage pouzet VRK formula (PVRK formula), which has the formula[4]:

$\bar{\emptyset}_{n}(t)=h \sum_{i=1}^{m} k\left(t, t_{n, i}, Y_{n, i}\right)$

With

$$
\begin{aligned}
& \quad Y_{n, i}= \\
& \bar{F}_{n}\left(t_{n, i}\right)+h \sum_{s=1}^{m} a_{i, s} k\left(t_{n}+c_{i} h, t_{n}+\right. \\
& \left.c_{s} h, Y_{n, s}\right), i=1, \ldots, m \quad \ldots(9)
\end{aligned}
$$

Here, the vectors $c=\left(c_{i}\right), b=\left(b_{i}\right)$ and the square matrix $A=\left(a_{i, s}\right)$ are completely determined by the Butcher array for ODEs [7]:

\begin{tabular}{|c|c|}
\hline $\mathrm{C}$ & $\mathrm{A}$ \\
\hline & $\mathrm{b}^{\mathrm{T}}$ \\
\hline
\end{tabular}

and $t_{n, i}=t_{n}+c_{i} h$. The lag term formula is given by:

$$
\bar{F}_{n}(t)=
$$

$f(t)+h \sum_{r=0}^{n-1} \sum_{s=1}^{m} b_{s} k\left(t, t_{r, s}, Y_{r, s}\right)$

...(10)

The following algorithm describe how to compute the approximate solution $y_{n+1}$ :

\section{Algorithm (PVRK): \\ Step(1):}

Evaluate the lag terms $\bar{F}_{n}\left(t_{n, i}\right), i=1, \ldots, m+1$, using the expression (10), with $c_{m+1}=1$.

\section{Step(2):}

Solving the nonlinear system (9) for $Y_{n, i} i=1, . ., m$.

\section{Step(3):}

Compute the increment term $\bar{\emptyset}_{n}\left(t_{n}+h\right)$ using (8).

\section{Step(4):}

Determine the approximate solution $y_{n+1}$ through the expression (7).

In this paper we use explicit PVRK of order two and four, that is, $\mathrm{m}=2$ and $\mathrm{m}=4$.

\section{Convergence results:}

For an easy analysis of the convergence of a PVRK method it is convenient to introduce the discrete increment operator $\emptyset_{n}$ associated with (8)-(10) and defined by:

$$
\emptyset_{n}\left[\bar{F}_{n}\right]\left(=\emptyset_{n}\left[\bar{F}_{n} ; t, h\right]\right)=
$$

$\emptyset_{n}(t), n=0, \ldots, N_{t}-1$

The following theorem holds[1].

Theorem(3): hold:

If the following assumptions

1. $\mathrm{A}=\left(\mathrm{a}_{\mathrm{ij}}\right), \quad \mathrm{b}=\left(\mathrm{b}_{\mathrm{i}}\right) \quad(\mathrm{i}, \mathrm{j}=1, \ldots, \mathrm{m})$ define an m-stage RK method of order $\mathrm{p}$ for a first order ODE.

2. the increment operator $\emptyset_{n}$ satisfies the Lipshitz condition

$$
\left|\emptyset_{n}\left[f_{n}\right]-\emptyset_{n}\left[g_{n}\right]\right| \leq
$$

$L\left|f_{n}-g_{n}\right|, t \in\left[t_{n}, T\right], n=0, \ldots, N_{t}-$ 1

for all real $f_{n}, g_{n}$ with $\mathrm{L}$ independent of $n$ and $h$.

Then the approximation $y_{n}$ generated by the PVRK method (7)-(10) satisfies for all sufficiently differentiable function $f$ and $k$ :

$$
\begin{aligned}
& \max _{1 \leq n \leq N_{t}}\left|y\left(t_{n}\right)-t_{n}\right|= \\
& O\left(h^{p}\right) \ldots(11)
\end{aligned}
$$

Theorem (3) used in [1]for the VIE of Hammerstein type and it is obvious that is true in general for nonlinear VIE's.

\section{Numerical Examples:}

Test Example(1):

Consider the following nonlinear VIE of the second type: 


$$
\begin{aligned}
y(x)=\sin x & +x \cos x+\frac{1}{3} x^{3}-x \\
& +\int_{0}^{x}\left(x y(t)-t^{2}\right) d t
\end{aligned}
$$

problem using MATLAB for which the analytical solution is $y(x)=\sin x$ over the interval $[0,1]$, with $\mathrm{N}_{\mathrm{t}}=10$, i.e. $\mathrm{h}=0.1$, and L.S.E=least squre error

Table (1) presents results from a computer program that solves this

Table (1): Exact and numerical solution for test example(1)

\begin{tabular}{|c|c|c|c|}
\hline $\mathbf{x}$ & exact & $\mathbf{R K 2}$ & $\mathbf{R K 4}$ \\
\hline $\mathbf{0}$ & 0.00000000000000 & 0.00000000000000 & 0.00000000000000 \\
\hline $\mathbf{0 . 1}$ & 0.09983341664683 & 0.09991674999753 & 0.09983313848725 \\
\hline $\mathbf{0 . 2}$ & 0.19866933079506 & 0.19884103090958 & 0.19866873060112 \\
\hline $\mathbf{0 . 3}$ & 0.29552020666134 & 0.29579319696598 & 0.29552472398958 \\
\hline $\mathbf{0 . 4}$ & 0.38941834230865 & 0.38981434554816 & 0.38944480460074 \\
\hline $\mathbf{0 . 5}$ & 0.47942553860420 & 0.47997660706173 & 0.47950749609751 \\
\hline $\mathbf{0 . 6}$ & 0.56464247339504 & 0.56539323769692 & 0.56483484772080 \\
\hline $\mathbf{0 . 7}$ & 0.64421768723769 & 0.64522847291802 & 0.64460060775820 \\
\hline $\mathbf{0 . 8}$ & 0.71735609089952 & 0.71870711807291 & 0.71803777978708 \\
\hline $\mathbf{0 . 9}$ & 0.78332690962748 & 0.78512387878655 & 0.78444545743552 \\
\hline $\mathbf{1}$ & 0.84147098480790 & 0.84385247029877 & 0.84319482964148 \\
\hline L.S.E & & $1.28826245473 \mathrm{e}-005$ & $4.878564082 \mathrm{e}-006$ \\
\hline
\end{tabular}

Test Example(2):

Consider the following nonlinear VIE of the second type:

$$
y(x)=x+\frac{1}{6} x^{3}+\int_{0}^{x}(t-x) y(t) d t
$$

Table(2) presents results from a computer program that solves this problem using MATLAB for which the analytical solution is $y(x)=x$ over the interval $[0,1]$, with $\mathrm{N}_{\mathrm{t}}=10$, i.e. $\mathrm{h}=0.1$.

Table(2): Exact and numerical solution for test example(2)

\begin{tabular}{|l|l|l|l|}
\hline $\mathbf{x}$ & exact & $\mathbf{R K 2}$ & $\mathbf{R K 4}$ \\
\hline $\mathbf{0}$ & 0.00000000000000 & 0.00000000000000 & 0.00000000000000 \\
\hline $\mathbf{0 . 1}$ & 0.10000000000000 & 0.09991656250000 & 0.09999951371528 \\
\hline $\mathbf{0 . 2}$ & 0.20000000000000 & 0.19983395833203 & 0.19999736302662 \\
\hline $\mathbf{0 . 3}$ & 0.30000000000000 & 0.29975426146225 & 0.29999217744187 \\
\hline $\mathbf{0 . 4}$ & 0.40000000000000 & 0.39967951683060 & 0.39998261683847 \\
\hline $\mathbf{0 . 5}$ & 0.50000000000000 & 0.49961171995287 & 0.49996738487211 \\
\hline $\mathbf{0 . 6}$ & 0.60000000000000 & 0.59955279701607 & 0.59994524194721 \\
\hline $\mathbf{0 . 7}$ & 0.70000000000000 & 0.69950458566557 & 0.69991501761959 \\
\hline $\mathbf{0 . 8}$ & 0.80000000000000 & 0.79946881667570 & 0.79987562230728 \\
\hline $\mathbf{0 . 9}$ & 0.90000000000000 & 0.89944709668660 & 0.89982605819210 \\
\hline $\mathbf{1}$ & 1.00000000000000 & 0.99944089217955 & 0.99976542920320 \\
\hline L.S.E & & $1.69427516218 \mathrm{e}-006$ & $1.124037744 \mathrm{e}-007$ \\
\hline
\end{tabular}

\section{Concluding remarks:}

In this paper we constructed VRK method of order two and four for a nonlinear VIE. A comparison is made between these methods 
depending on least square error (L.S.E.), which is calculated from the numerical solution against the exact solution.

In general, methods which are used in this paper proved their effectiveness in solving a nonlinear VIE numerically and finding accurate results, we noticed that the fourth order VRK methods give the best approximation to solve nonlinear VIE.

The methods presented in this paper are highly parallelizable and we think that they can be extended in a natural way to system of VIEs.

\section{References:}

1. I. Del Prete, 2005, Efficient numerical methods for Volterra integral equations of hammerstein type, $\mathrm{PhD}$ thesis, Universiy of $\mathrm{Di}$ Napli Federico II, Italy.

2. T. Diogo, P. Lima and M. Rebelo, 2005, Computational methods for a nonlinear Volterra integral equations, Proceedings of Hercma, 100-107.

3. P. Darania, A. Ebadian and A. V. Oskoi, 2006, Linearization method for solving nonlinear integral equations, Hindawi Publishing Corporation, Mathematical Problems in Engineering, V2006, Article ID 73714, P1-10.

4. G. Capobianco, D. Conte, I. Dell Prete and E. Russo, 2007, Fast Runge-Kutta methods for nonlinear convolution systems of Volterra integral equations, BIT, V(47), pp 259-275.

5. T. Diogo and P. Lima, 2007, Collocation solutions of a weakly singular Volterra integral equation, TEMA Tend Mat. Apl. comput., 8(2), pp 229-238.

6. G. Capobianco, D. Conte, I. Del Prete and E. Russo, 2008, Stability analysis of fast numerical methods for Volterra integral equations, Electronic Transactions on Numerical Analysis, V(30), pp 305322.

7. M. K. Jain, 1979, Numerical solution of Differential equations, John Wiley\& Sons, Inc., New York, pp 443.

\section{طرق فولتيرا راتجا_كوتا لحل معادلات فولتيرا التكاملية اللاخطية}

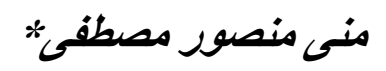

$$
\text { ققسم الرياضيات, كلية العلوم للبنات, جامعة بغداد, الجادرية, بغداد, العر اق }
$$

في هذا البحث طرق فولتيرا رانجا-كوتا التي تتضمن طرق من الرتبة الثانية والرابعة طبقت على معادلات

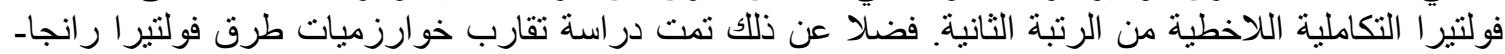

و كوتا. 